\title{
INTRODUCTION TO BIOINFORMATICS, MEDICAL INFORMATICS AND THE LAW
}

This volume covers a broad range of legal topics relating to the fields of bioinformatics and medical informatics. While the precise meaning of these terms is not generally agreed, as A. James Cuticchia aptly observes in Chapter 1, the topic, broadly stated, concerns the intersection of biomedical information and computer programming within the contexts of scientific research, product development, and healthcare delivery. As such, a number of usually distinct bodies of legal doctrine come together in this area, sometimes overlapping, sometimes colliding in unexpected ways.

Part I of this volume offers an overview of the current landscape of bioinformatics and medical informatics. Chapter 1 provides the reader with a broad overview of these two related fields, describing the medical and technological realms in which they are increasingly crucial. In Chapter 2, Janis Geary, Mary Majumder, Jill Oliver Robinson, Christi Guerrini, and Robert Cook-Deegan place these technologies within the broader social construct of "medical information commons"-public, semi-public and private aggregations of medical, health and research data that are increasingly being produced around the world. The authors, in surveying these new and potentially valuable knowledge commons, also note the unique challenges that they pose in terms of governance, privacy, and marginalized populations.

Part II turns to the legal issues arising from the development and acquisition of informatics tools for use in a laboratory or healthcare setting. In Chapter 3, Brett Lockwood offers practical advice regarding the licensing of software for informatics applications, covering both custom-designed and off-the-shelf commercial software. In Chapter 4, Michele Herman discusses the use of open source software in informatics applications, including the range of available licenses, the impact of "copy-left" provisions, requirements for attribution, non-commercial use and limitations on derivative works. And in Chapter 5, Heather Meeker covers the commercial development of software for informatics applications, both internally and through third party contractors.

Part III addresses developments in patent and innovation law that are important for informatics applications. In Chapter 6, Gregory Kirsch and Charley Brown observe that legal landscape for software patents has changed dramatically since 2013. In 2014, the US Supreme Court handed down its landmark decision in Alice Corp. $v$ CLS Bank, which altered the patent-eligibility requirements for computer-implemented inventions. Since then, the US Court of Appeals for the Federal Circuit has further refined the tests for patentability set forth by the Supreme Court and further evolved the types of inventions that are deemed to be patent-eligible. In Chapter 7, Saurabh Vishnubhakat and Arti Rai examine the impact of these changes on patent office practice and patents issued in the United States. 
Part IV turns to the institutions and collaborative arrangements in which informatics applications are developed and used today. In Chapter 8, Jeff Carter-Johnson, Jennifer Carter-Johnson, and Jorge Contreras discuss technology development and transfer within academic institutions, including the provisions of the Bayh-Dole Act of 1980, as well as more recent academic research issues such as the Nine Points accord and IP license guidelines and best practices issued, among others, by the US National Institutes of Health. In Chapter 9, Jorge Contreras and Adrian Thorogood address the collaborative development of interoperability standards for bioinformatics data and systems, with a focus on electronic medical records. The legal issues covered include both copyright and patent issues endemic to technical standardization, particularly as they have emerged over the past decade in the wireless telecommunications and computer networking industries.

Part $\mathrm{V}$ then turns to the host of data protection and privacy issues applicable to informatics applications in the US and Europe. In Chapter 10, Jon Garon discusses issues arising from the creation, use and licensing of biomedical data and databases, including the divergent legal status of databases in the US and Europe. In Chapter 11, Jorge Contreras summarizes the history of US federal agency policies relating to the sharing of scientific data, particularly in the areas of genomics as well as significant policies promulgated by public-private partnerships and the private sector. In Chapter 12, Lucy Thomson and Arthur Peabody cover the many issues arising with respect to patient data privacy and security in the area of bioinformatics, including the Health Insurance Portability and Accountability Act (HIPAA), the Common Rule, the Health Information Technology for Economic and Clinical Health (HITECH) Act, the Genetic Information Nondiscrimination Act (GINA), and the National Institutes of Health (NIH) Policy for Data Management and Sharing, as well as numerous state medical records and data breach regulations. Chapter 13 turns toward the European Union, as Christopher Jones explores the emerging issues for informatics under the EU General Data Protection Regulation (GDPR). And in Chapter 14, by Leslie Francis and John Francis explore the use of informatics applications and data for public health modeling and its legal and privacy implications.

While no single work can cover the entire set of legal issues arising from large, dynamic, and complex fields such as bioinformatics and medical informatics, this volume strives to offer the reader insight into some of the major legal trends and considerations applicable to these fields today. 
Jorge L. Contreras, A. J. Cuticchia, and Gregory J. Kirsch - 9781839105951 Downloaded from PubFactory at $\odot 4 / 26 / 2023$ 02: $01: 25 \mathrm{AM}$ via free access 